\title{
A PRODUÇÃO DO CONHECIMENTO EM ENFERMAGEM E ENVELHECIMENTO: ESTUDO BIBLIOMÉTRICO
}

\author{
Ana Paula Xavier Ravelli, Gisele Cristina Manfrini Fernandes², Sayonara de Fátima Faria Barbosa, Eunice \\ Simão ${ }^{4}$, Silvia Maria Azevedo dos Santos ${ }^{5}$ Bettina Horner Schlindwein Meirelles ${ }^{6}$
}

\footnotetext{
${ }^{1}$ Doutoranda pelo Programa de Pós-Graduação em Enfermagem (PEN) da Universidade Federal de Santa Catarina (UFSC). Professor Assistente na Universidade Estadual de Ponta Grossa. Paraná, Brasil. E-mail: anapxr@hotmail.com

${ }^{2}$ Doutoranda do PEN/UFSC. Bolsista CNPq. Santa Catarina, Brasil. E-mail: gisamanfrini@yahoo.com.br

${ }^{3}$ Doutora em Ciências da Saúde. Professor Adjunto do Departamento de Enfermagem da UFSC. Santa Catarina, Brasil. E-mail: sayonara@ccs.ufsc.br

${ }^{4}$ Doutoranda do PEN/UFSC. Enfermeira da Secretaria de Estado de Saúde de Santa Catarina. Santa Catarina, Brasil. E-mail: eunice@saude.sc.gov.br

${ }^{5}$ Doutora em Educação. Professor Adjunto do Departamento de Enfermagem e do PEN/UFSC. Santa Catarina, Brasil. E-mail: azevedosm@matrix.com.br

${ }^{6}$ Doutora em Enfermagem. Professor Adjunto do Departamento de Enfermagem e do PEN/UFSC. Santa Catarina, Brasil. E-mail: betinam@nfr.ufsc.br
}

RESUMO: As bases de dados eletrônicas são ferramentas promotoras de conhecimento e possibilitam o desenvolvimento científico e tecnológico na área da saúde e da enfermagem. Este estudo bibliométrico teve como objetivo mapear os artigos originais sobre enfermagem e envelhecimento, publicados no período de 2003 a 2008 na base de dados SciELO, Brasil. Foram selecionados 115 artigos, analisados descritivamente quanto à formação, titulação e atuação dos autores, às instituições representadas nos artigos, aos periódicos em que foram publicados e aos descritores mais citados. Os resultados apontam uma significativa participação de enfermeiros, doutores e docentes no desenvolvimento de estudos. Há predomínio nas publicações de instituições de ensino e pesquisa provenientes da região Sudeste. Os resultados evidenciam a importância desses centros de pesquisa e de divulgação do conhecimento.

DESCRITORES: Tecnologia. Envelhecimento. Enfermagem. Conhecimento.

\section{KNOWLEDGE PRODUCTION IN NURSING AND AGING: A BIBLIOMETRIC STUDY}

\begin{abstract}
Electronic databases are knowledge promotion tools and allow the scientific and technological development in nursing and health. The objective of this bibliometric study was to map original articles on nursing and aging, published from 2003 to 2008 in the Brazilian SciELO database. 115 articles were selected and then analyzed descriptively concerning the authors' formal education, degree attained, and activities, as well as the institutions represented in articles, the journal where the papers had been published, and the most cited keywords. The results point to a significant participation among nurses, physicians, and professors in the development of studies. There was predominance of education and research institutions and research in the publications which originated from the southeastern region of Brazil. The results evidence the importance of these research centers and knowledge dissemination on this subject.
\end{abstract}

DESCRIPTORS: Technology. Aging. Nursing. Knowledge.

\section{LA PRODUCIÓN DEL CONOCIMIENTO EN ENFERMERÍA Y EL ENVEJECIMIENTO: ESTUDIO BIBLIOMÉTRICO}

RESUMEN: Las bases de datos electrónicas son herramientas para promover el conocimiento y permitir el desarrollo científico y tecnológico en el área de la salud y de la enfermería. Este estudio es bibliométrico y su objetivo es mapear los artículos originales de enfermería sobre el envejecimiento, publicados en el período de 2003 a 2008, en la base SciELO. Se seleccionaron 115 artículos, analizados descriptivamente en relación a: la formación, el rendimiento y la valoración de los autores, las instituciones representadas en los artículos, los periódicos en que fueron publicados y los descriptores más citados. Los resultados muestran una significativa participación de los enfermeros, doctores y profesores en el desarrollo de esos estudios. En las publicaciones hay un predominio de instituciones de enseñanza e investigación de la región sudeste del Brasil. Los resultados evidencian la importancia de esos centros en la investigación y divulgación del conocimiento.

DESCRIPTORES Tecnología. Envejecimiento. Enfermería. Conocimiento. 


\section{INTRODUÇÃO}

Os avanços da ciência e da tecnologia têm trazido significativas mudanças no processo de viver humano, no qual agregam repercussões importantes para o mundo cotidiano do trabalho, da educação, da pesquisa e da saúde. Ao pensarmos no ciclo de vida humano, do nascimento ao envelhecimento e à morte, torna-se imprescindível reconhecer as implicações da ciência e da tecnologia às perspectivas atuais do cuidado à saúde.

Reportando-se ao processo de envelhecer, antes considerado um fenômeno e hoje fazendo parte da sociedade, evidencia-se que "no Brasil, nas últimas décadas tem-se verificado um aumento da população idosa, devido a um decréscimo das taxas de natalidade e mortalidade, e a um aumento da expectativa de vida" .1:449

Destacam-se nesse momento as políticas de saúde voltadas ao processo de envelhecer, como a Política Nacional de Saúde da Pessoa Idosa ${ }^{2}$ com a Portaria GM No 2.528, de 19 de outubro de 2006, e a Portaria No 648, de 28 de março de 2006. Também no final da década de 90, a Organização Mundial da Saúde passou a utilizar o conceito de envelhecimento ativo buscando incluir, além dos cuidados com a saúde, outros fatores que afetam o envelhecimento. ${ }^{2-3}$

A ciência e a tecnologia constituem os meios para a formação de profissionais enfermeiros cuidadores e também pesquisadores, o que contribui para a produção e ampla disseminação do conhecimento. As bases de dados eletrônicas são ferramentas promotoras dessa troca de conhecimentos entre os pesquisadores, de forma a proporcionar novas possibilidades de desenvolvimento científico e tecnológico.

A importância dos periódicos na comunicação científica foi reforçada com a criação das bases de dados, que passaram a exercer o papel não só de reuni-las, mas também de avaliá-las e selecioná-las. ${ }^{3}$ E essas bases de dados que se tornaram disponíveis eletronicamente por meio da internet ampliaram o seu potencial de utilização pelos pesquisadores.

No Brasil, a base de dados Scientific Electronic Library Online (SciELO) é um modelo para a publicação eletrônica cooperativa de periódicos científicos na internet. Especialmente elaborado para responder às necessidades da comunicação científica nos países em desenvolvimento - particularmente na América Latina e no Caribe -, propõe-se a disponibilizar, em texto integral, artigos e periódicos completos para aumento da visibilidade e acesso - Open Access - à ciência brasileira e regional. Trata-se também de uma resposta à necessidade de soluções confiáveis para a publicação eletrônica de periódicos e que sejam compatíveis com as iniciativas internacionais mais importantes. ${ }^{4}$

Além disso, atende a uma antiga demanda referente à operação de bases de dados bibliográficos para não apenas controlar e disseminar a literatura científica, mas também permitir a produção de indicadores para subsidiar estudos de bibliometria, informetria e cienciometria sobre a produção científica nacional relevante. ${ }^{5}$

A bibliometria vem sendo utilizada nas diversas áreas do conhecimento como metodologia para a obtenção de indicadores de avaliação da produção científica. De forma geral, o princípio da bibliometria é analisar a atividade científica ou técnica pelo estudo quantitativo das publicações e o seu principal objetivo é o desenvolvimento de indicadores cada vez mais confiáveis. ${ }^{6}$

A pesquisa bibliométrica apóia-se em estudos realizados em bases de dados bibliográficas, indexadores e resumos, em diretórios e catálogos de títulos de periódicos e em referências e citações. Entre outros, seu campo de aplicação mais frequente - e mais relevante para este trabalho - é a identificação das características temáticas da literatura. Em síntese, a pesquisa bibliométrica se dá através do estudo dos aspectos quantitativos da produção, disseminação e uso da informação publicada. ${ }^{7-8}$

A importância dos estudos bibliométricos é sustentada pela necessidade de conhecer e avaliar a produtividade e a qualidade da pesquisa dos atores (autores/pesquisadores), permitindo a detecção de modelos de dispersão e padrões de comportamento de citações em sua produção científica. ${ }^{3}$ Tais modelos e padrões de comportamento ajudam a entender como o conhecimento científico é difundido e incorporado entre os atores e seus pares, bem como entre o público em geral. ${ }^{9}$ Ainda, pode-se afirmar que os estudos bibliométricos têm sido cada vez mais requisitados e utilizados para a quantificação da produção e também para outras finalidades, como identificar grupos e áreas de excelência acadêmica. ${ }^{10}$ Assim, este artigo destacará o envelhecimento e o que está sendo produzido e publicado nessa temática, tendo como instrumento alguns indicadores bibliométricos. 
Desse modo, o presente estudo teve por objetivo mapear os artigos originais sobre enfermagem e envelhecimento, publicados no período de 2003 a 2008, na base de dados SciELO, Brasil.

\section{METODOLOGIA}

Este estudo é do tipo bibliométrico e descritivo, e nele utilizou-se a SciELO, que contempla uma coleção selecionada de periódicos científicos Ibero-americanos. O objetivo da SciELO é o desenvolvimento de uma metodologia comum para preparação, armazenamento, disseminação e avaliação da produção científica em formato eletrônico. Entre seus objetivos específicos está a produção de indicadores bibliométricos. ${ }^{11}$

Nesta pesquisa, a seleção das publicações foi feita a partir de critérios de inclusão e de exclusão. Nesse sentido, apropriou-se das publicações de artigos originais utilizando o descritor enfermagem, associado aos descritores: envelhecimento, envelhecimento da população, idoso, saúde do idoso, advindos dos Descritores em Ciência da Saúde (DeCS) sob consulta. Também foram utilizadas as palavras envelhecimento humano, envelhecimento populacional, envelhecimento saudável, idosos e senilidade na estratégia de localização das publicações. A pesquisa compreendeu os artigos publicados no período de janeiro de 2003 a julho de 2008, e a busca e a coleta dos dados foram realizadas nos meses de agosto e setembro de 2008. Os critérios de exclusão foram todos os demais tipos de publicação (editoriais, comentários, reflexão, relato de experiência e revisão da literatura) e os artigos originais que não apresentavam algum dos descritores acima citados.

Os artigos selecionados foram analisados conforme dados bibliométricos relativos a: formação profissional, titulação acadêmica, área e local de atuação dos autores, bem como periódicos em que os artigos foram publicados e descritores mais utilizados. Os dados foram obtidos a partir do acesso a cada um dos artigos e, simultaneamente, organizados em um instrumento no formato de tabela, com os respectivos campos: ano de publicação, periódico, título do artigo, nome dos autores, formação, titulação e instituição representante, descritor (es). Os dados referentes a formação profissional, titulação acadêmica (estudante, doutorado, mestrado ou pós-doutorado) e área de atuação (assistencial, docente, outro) foram coletados diretamente nos artigos ou através de consulta no currículo Lattes dos autores, pelo link Curriculum ScienTI da página dos artigos na SciELO. Considerou-se um dado não informado do autor quando este não estava disponibilizado no seu currículo Lattes.

\section{RESULTADOS E DISCUSSÃO}

Foram identificados 154 artigos a partir dos descritores selecionados e, com a utilização dos critérios de exclusão, foram eliminados 39 artigos. Desse modo, a amostra do estudo contou com 115 artigos para análise.

Quanto à formação profissional, titulação acadêmica e área de atuação profissional dos autores, identificaram-se pesquisadores de diferentes áreas do conhecimento. Dos autores com formação na área da saúde destacaram-se significativamente os enfermeiros (60\%), seguidos dos médicos $(6 \%)$ e dos psicólogos (5\%). Entre as outras formações profissionais havia assistente social, advogado, biólogo, dentista, engenheiro, físico, estatístico, farmacêutico matemático, fonoaudiólogo, fisioterapeuta, educador físico, nutricionista, sociólogo, matemático e terapeuta ocupacional. Também foram identificados autores com mais de uma formação na área da saúde ou em outra área. A Tabela 1 mostra a distribuição dos autores conforme a formação profissional.

\section{Tabela 1 - Formação profissional dos autores dos artigos publicados no período de 2003 a 2008}

\begin{tabular}{lrr}
\hline Profissão & $\mathbf{n}$ & \% \\
\hline Enfermeiro & 178 & 60 \\
Médico & 18 & 6 \\
Farmacêutico & 6 & 2 \\
Dentista & 6 & 2 \\
Psicólogo & 16 & 5 \\
Outros & 84 & 20 \\
Não informado & 16 & 5 \\
\hline Total & $\mathbf{3 2 4}$ & $\mathbf{1 0 0}$ \\
\hline
\end{tabular}

Quanto aos resultados sobre a titulação dos autores dos artigos, identificou-se que 50\% têm grau de doutorado. Não foram considerados dados de caracterização quanto à formação e titulação dos estudantes de pós-graduação. Entretanto, foi observada uma expressiva participação de estudantes, tanto de nível de graduação quanto de pós-graduação, evidenciando-se, de certa forma, a vinculação da produção acadêmica aos cursos de pós-graduação strictu sensu e iniciação científica. A Tabela 2 apresenta o perfil dos autores quanto à titulação acadêmica. 
Tabela 2 - Titulação dos autores dos artigos publicados no período de 2003 a 2008

\begin{tabular}{lrr}
\hline Titulação & $\mathbf{n}$ & $\mathbf{\%}$ \\
\hline Aluno de graduação & 32 & 10 \\
Aluno de pós-graduação & 56 & 17 \\
Profissional com mestrado & 32 & 10 \\
Profissional com doutorado & 162 & 50 \\
Profissional com pós-doutorado & 18 & 5 \\
Não informado & 24 & 8 \\
\hline Total & $\mathbf{3 2 4}$ & $\mathbf{1 0 0}$ \\
\hline
\end{tabular}

No que se refere à área de atuação dos autores, verificou-se que, embora haja maior concentração de docentes (82\%) no desenvolvimento de estudos e publicações sobre a temática do envelhecimento, chama-nos a atenção a participação de profissionais que atuam na assistência $(12 \%)$ e que representam serviços de saúde vinculados ou não às instituições de ensino e pesquisa. Houve autores que se identificaram como atuantes em cargos técnico-administrativos (1\%), e não se obteve informação sobre a área de atuação de 5\% dos autores analisados. Essa falta de informação nos artigos ou indisponibilidade de currículo Lattes de muitos dos autores, expressa um fator dificultador para essa bibliometria. Por outro lado, evidencia a importância de inclusão de dados completos (e padronizados) em todos os periódicos disponibilizados em base de dados, de modo a facilitar o desenvolvimento de estudos bibliométricos mais vastos.

Quanto aos autores dos artigos com formação de enfermeiro, notou-se que estes se apresentam em seis diferentes situações de atuação, relacionadas à titulação acadêmica: docente doutor, docente mestre, docente pós-doutor, assistencial com mestrado, somente assistencial e somente docente. Cabe informar que não foram considerados os dados relativos à titulação de pós-graduação latu sensu. Dos 175 autores enfermeiros, $61,1 \%$ são docentes doutores, $17,1 \%$ são docentes mestres, $4,5 \%$ são docentes pósdoutores e 7,2\% são assistenciais com mestrado.

Os autores da amostra de artigos selecionados representam 62 instituições, entre universidades públicas, privadas e estrangeiras, instituições assistenciais públicas e privadas, secretarias municipais de saúde. Entre essas instituições, observou-se um predomínio de pesquisas realizadas em universidades públicas, especialmente da região Sudeste.

Houve artigos que representaram mais de uma instituição, especialmente aqueles elaborados por autores vinculados a cursos de pós-graduação, e também artigos publicados entre instituição de ensino-pesquisa e serviço de saúde. A maioria dos artigos foi elaborada por pesquisadores de instituições de ensino e pesquisa (87\%), embora uma parcela importante tenha sido elaborada por pesquisadores com vínculos em instituições assistenciais (13\%).

Entre as instituições assistenciais representadas pela amostra analisada neste estudo bibliométrico, os hospitais universitários, públicos e privados têm relevância na participação da produção científica. Na análise de outro estudo de revisão, as publicações sobre enfermagem e envelhecimento parecem manter o paradigma hospitalocêntrico norteando os cenários das investigações em Enfermagem. ${ }^{12}$

A maioria das instituições de pesquisa está localizada na região Sudeste. Em seguida aparecem as regiões Sul e Nordeste. Esses resultados dão subsídios para o reconhecimento do desenvolvimento de pesquisas voltadas à formação de recursos humanos na área, bem como para a identificação de tendências.

Tabela 3 - Regiões e instituições mais representadas nos artigos publicados no período de 2003 a 2008

\begin{tabular}{llc}
\hline Região & \multicolumn{1}{c}{ Instituição } & Número de artigos \\
\hline \multirow{3}{*}{ Sul } & Universidade Federal de Santa Catarina & 8 \\
& Universidade Federal do Rio Grande do Sul & 6 \\
& Universidade Estadual de Maringá & 5 \\
& & 25 \\
Sudeste & Universidade de São Paulo/SP & 17 \\
& Universidade de São Paulo/RP & 10 \\
Nordeste & Universidade Estadual do Rio de Janeiro & 9 \\
\hline
\end{tabular}


Outra observação que pode ser ressaltada é que há publicações feitas por participação conjunta de instituições universitárias nacionais e três estrangeiras, as quais representam países como Portugal, Espanha e Canadá. Um resultado interessante trata do tipo de instituição assistencial ou tipo de serviço de saúde, em que 11 artigos são identificados pelos autores como Programa Saúde da Família, Secretaria Municipal de Saúde e Centro de Atenção e 12 artigos representados por instituição hospitalar - destes, seis são hospitais universitários.

Quanto aos periódicos em que os artigos foram publicados no período de 2003 a 2008, podese verificar uma distribuição maior na Texto \& Contexto Enfermagem (24,3\%), na Revista LatinoAmericana de Enfermagem (15,6\%) e na Revista da Escola de Enfermagem da USP (14\%). A tabela abaixo mostra a distribuição, por periódico, do número de artigos publicados a cada ano.

Tabela 4 - Número de artigos publicados anualmente por periódico, no período de 2003 a 2008

\begin{tabular}{lccccccc}
\hline Periódico & $\mathbf{2 0 0 3}$ & $\mathbf{2 0 0 4}$ & $\mathbf{2 0 0 5}$ & $\mathbf{2 0 0 6}$ & $\mathbf{2 0 0 7}$ & $\mathbf{2 0 0 8}$ & Total \\
\hline Acta Paulista de Enfermagem & - & - & 3 & 5 & 3 & 1 & 12 \\
Arquivo Brasileiro de Cardiologia & - & 1 & - & - & - & - & 1 \\
Arquivo Brasileiro de Endocrinologia Metabólica & - & 1 & - & - & - & - & 1 \\
Cadernos de Saúde Pública & 1 & 1 & - & 1 & 1 & 1 & 5 \\
Ciência e Saúde Coletiva & - & 1 & - & 2 & 4 & 3 & 10 \\
Interface & - & - & - & - & 1 & - & 1 \\
Physis & - & - & 1 & - & 1 & - & 2 \\
Revista Brasileira de Enfermagem & - & - & 1 & - & 2 & 3 & 6 \\
Revista Brasileira de Epidemiologia & - & - & 2 & 1 & 2 & - & 5 \\
Revista Brasileira de Medicina do Esporte & - & - & - & - & - & 1 & 1 \\
Revista da Escola de Enfermagem da USP & - & - & 2 & 3 & 7 & 4 & 16 \\
Revista Latino-Americana de Enfermagem & 3 & 2 & 3 & 5 & 4 & 1 & 18 \\
Texto \& Contexto Enfermagem & - & - & 3 & 3 & 9 & 13 & 28 \\
Revista de Saúde Pública & - & 1 & 1 & 1 & 1 & 2 & 6 \\
Saúde e Sociedade & - & - & - & - & 2 & 1 & 3 \\
\hline Total & $\mathbf{4}$ & $\mathbf{7}$ & $\mathbf{1 6}$ & $\mathbf{2 1}$ & $\mathbf{3 7}$ & $\mathbf{3 0}$ & $\mathbf{1 1 5}$ \\
\hline
\end{tabular}

Cabe ressalvar que a Texto \& Contexto Enfermagem é um periódico que apresenta números temáticos, o que justifica também o grande número de artigos sobre envelhecimento identificados nela, especialmente nos últimos dois anos (esse foi o tema, por exemplo, do segundo número de 2008, que incluiu artigos sobre a atenção à saúde do idoso e que configura uma importante concentração de estudos recentes sobre a temática).

Partindo da observação sobre a ocorrência de publicações com o tema envelhecimento, referencia-se um estudo de revisão, realizado na base de dados LILACS e nas dissertações catalogadas no acervo da Escola de Enfermagem de Ribeirão
Preto, da Universidade de São Paulo, sobre publicações do período de 1980 a 2000, que constatou predominância de estudos com enfoque geriátrico, ou seja, sobre aspectos físicos e biológicos do idoso. Porém, outro resultado foi que, no último intervalo, de 1995 a 2000, o enfoque gerontológico é que foi maior em relação ao geriátrico, relacionando-se mais às pesquisas demográficas e epidemiológicas que ressaltam a importância de investigar o tema idoso no contexto social e cultural. ${ }^{13}$

Nesse sentido, pretende-se sinalizar aqui que tais perspectivas, tanto geriátricas como gerontológicas, abordadas nos estudos revisados, apontam os interesses de investigação da enfermagem 
na temática do idoso, inclusive pensando com o passar dos anos e sua relação com a preocupação de desenvolvimento científico da área. Para os autores, a ocorrência das publicações expressa perspectivas de pesquisa da enfermagem sobre a velhice no país, suas representações e lacunas, de acordo com os acontecimentos que dão origem às necessidades e interesses de investigação. Todavia, o aumento de pesquisas voltadas às diferentes dimensões nas questões do envelhecimento enfatiza a necessidade de se dedicar mais atenção ao enfoque gerontológico.

Tendo em vista a estratégia de busca dos artigos na base de dados SciELO a partir de descritores do DeCS relacionados à enfermagem e ao envelhecimento, os quais constituíram um dos principais critérios de inclusão neste estudo bibliométrico, optou-se por analisar também a frequência desses descritores na amostra de artigos selecionada.

Assim, dos 115 artigos analisados, totalizaram-se 147 descritores no geral. Destes, os mais utilizados foram: idoso (47\%), saúde do idoso $(20,4 \%)$, envelhecimento $(19 \%)$ e enfermagem $(13,6)$. Um levantamento bibliográfico sobre enfermagem e envelhecimento restrito à Revista Brasileira de Enfermagem, contemplando o período de 2000 a 2006, mostrou que, pesquisas referentes ao envelhecimento denotam uma pequena produção científica de enfermagem referente ao idoso, considerando-se outras temáticas investigadas nesse mesmo período. ${ }^{6}$

Ainda que as mudanças demográficas e populacionais tenham dado maior ênfase às necessidades de pesquisa na área do envelhecimento, vê-se que a enfermagem tem, nos últimos anos, desenvolvido estudos mais específicos sobre a temática, visto o significativo número de pesquisadores doutores e pós-graduandos.

Ainda quanto aos resultados referentes à frequência dos descritores, destaca-se a situação do descritor enfermagem nesta pesquisa, chamandonos a atenção para a importância de os pesquisadores identificarem com tal descritor os trabalhos desenvolvidos na enfermagem, visto que, embora $68 \%$ dos pesquisadores analisados sejam enfermeiros, a expressividade de suas publicações com o descritor enfermagem é pequena (10,2\%). Isso também nos leva a pensar na questão da qualificação e representatividade da publicação científica da enfermagem para a ciência de modo geral.

No entanto, outro estudo identificou que títulos de artigos de um determinado periódico científico da área da saúde nem sempre apresentam os termos necessários a uma recuperação eficaz da informação, considerando que os resultados de sua análise evidenciaram um número bem maior de termos considerados diferentes, ressaltando a idéia de que os títulos dos artigos de um periódico científico nem sempre representam os assuntos dos artigos. ${ }^{14}$

Sendo assim, nos tempos atuais, marcados por intenso uso de tecnologias eletrônicas de organização e recuperação de informações, há a necessidade de que o título de artigo de periódico científico, na área de saúde e nas demais áreas especializadas, seja claro, conciso e preciso, constando de termos que possam servir como palavraschave significativas, de modo a facilitar inclusive o processo de indexação das publicações.

Portanto, um estudo realizado em 2004 indicou que as Escolas de Enfermagem do Brasil apresentam escasso número de docentes envolvidos em programas de mestrado ou doutorado, desenvolvendo pesquisas ou estudando enfermagem gerontológica, baseando o ensino de graduação, especialmente, sob temas vinculados à experiência ou conhecimentos gerais do professor. ${ }^{15}$ Entretanto, os dados obtidos no presente estudo apontam uma forte qualificação dos pesquisadores envolvidos com essa temática, o que pode indicar um crescimento na participação de docentes na realização de pesquisa na área de saúde do idoso.

\section{CONCLUSÕES}

O estudo aponta tendências para o desenvolvimento de pesquisas na área da enfermagem e do envelhecimento, assim como o envolvimento de alunos de graduação e de pós-graduação na publicação de artigos sobre o tema, o que expressa também a direção da formação de profissionais e pesquisadores atentos à temática.

Quanto às perspectivas de pesquisa, o passar dos anos mostra que os interesses e focos de investigação sobre a temática do envelhecimento na Enfermagem e na Saúde expressam, de certa forma, o próprio desenvolvimento da área da gerontologia e geriatria, uma vez que os acontecimentos históricos e atuais a respeito da demografia, epidemiologia e modo de vida das pessoas idosas se tornam necessidades para o conhecimento científico e a discussão desses fenômenos em outras áreas. ${ }^{7}$

As análises bibliométricas podem se configurar em uma metodologia tecnológica importante para a enfermagem, por desvelar padrões de pesquisa e identificação de tendências. Podem 
também ser utilizadas em qualquer base de dados, sendo sugeridos novos estudos.

O uso dos recursos disponíveis na base de dados SciELO se mostrou interessante para a realização de estudos desse tipo em curto espaço de tempo, além de propiciar a possibilidade de estudos posteriores com análise de outros indicadores. A contribuição desse estudo para a Enfermagem se deve aos indicadores resultantes quanto às instituições de pesquisa e aos periódicos que mais se destacam na produção do conhecimento sobre o tema em nível nacional. Sugerem-se outras iniciativas de ampliação de busca por artigos sobre o tema enfermagem e envelhecimento na base SciELO em relação à abrangência regional (América Latina e Caribe), de modo a se obter interessantes resultados sobre o perfil bibliométrico das publicações a respeito do tema.

\section{REFERÊNCIAS}

1. Rocha FCV, Brito CMS, Luz MHBA, Figueiredo MLF. Análise da Produção Científica sobre o Idoso na REBEn. Rev Bras Enferm. 2007 Jul-Ago; 60(4):449-51.

2. Ministério da Saúde (BR). Secretaria de Atenção à Saúde. Departamento de Atenção Básica. Cadernos de Atenção Básica - N 19: envelhecimento e saúde da pessoa idosa. Brasilia (DF): MS, 2006.

3. Mugnaini R. Caminhos para adequação da avaliação da produção científica brasileira: impacto nacional versus internacional [tese]. São Paulo (SP): Universidade de São Paulo; 2006 [acesso 2008 Nov 11]. Disponível em: http://www.teses.usp.br/ teses/disponiveis/27/27151/tde-11052007-091052

4. Packer AL, Bijone MR, Arati A, Takenaka RM, García AP, Silva AC et al. SciELO: uma metodologia para publicação eletrônica. Ci Inf. 1998 Mai-Ago; 27(2):109-21.

5. Santos ASR, Souza PA, Valle AMD, Cavalcanti ACD, Sá SPC, Santana RF. Caracterização dos diagnósticos de enfermagem identificados em prontuários de idosos: um estudo retrospectivo. Texto Contexto Enferm. 2008 Jan-Mar; 17(1):141-9.
6. Santos, RN. (2003). Produção científica: por que medir? O que medir? RDBCI. 2003 1(1):22-38.

7. Araújo RJA, Arencibia JR. Informetría, bibliometría y cienciometría: aspectos teórico-prácticos. ACIMED [online]. 2002 Ago [acesso 2008 Dez 15]; 10(4):5-6. Disponível em: http://scielo.sld. $\mathrm{cu} /$ scielo.php? script=sci_arttext\&pid=S102494352002000400004\&lng=es\&nrm=iso

8. Vanti N. Da bibliometria à webometria: uma exploração conceitual dos mecanismos utilizados para medir o registro da informação e a difusão do conhecimento. Ci Inf. 2002; 31(2):152-62.

9. Carvalho LF. Bibliometria e saúde coletiva: análise dos periódicos Cadernos de Saúde Pública e Revista de Saúde Pública [dissertação]. Rio de Janeiro (RJ): Escola Nacional de Saúde Pública Sérgio Arouca; 2005.

10. Filippo DFMT. Bibliometría: importancia de los indicadores bibliométricos. In: Albornoz M. editor. El estado de la ciencia: principales indicadores de ciencia y tecnología Iberoamericanos / interamericanos. Buenos Aires (AR): Artes Gráfica Integradas, 2002.

11. Goldenberg S, Castro RCF, Azevedo FRM. Interpretação dos dados estatísticos da SciELO (Scientific Eletronic Library Online). Acta Cir Bras [online] 2007 Jan-Fev [acesso 2008 Dez 15]; 22(1):1-7. Disponível em: http://www.scielo.br/pdf/acb/ v22n1/01.pdf.

12. Rocha FCV, Brito CMS, Luz MHBA, Figueiredo MLF. Análise da produção científica sobre o idoso na REBEn. Rev Bras Enferm, 2007 Jul-Ago; 60(4):449-51.

13. Freitas MC, Maruyama SAT, Ferreira TF, Motta AMA. Perspectivas das pesquisas em gerontologia e geriatria: revisão da literatura. Rev Latino-am Enfermagem. 2002 Mar-Abr; 10(2):221-8.

14. Duarte E. Comparação entre termos de indexação e palavras dos títulos dos artigos do periódico "Cadernos de Saúde Pública (2000/2005)" [dissertação]. Belo Horizonte (MG): Universidade Federal de Minas Gerais. Programa de PósGraduação em Ciência da Informação; 2007.

15. Diogo MJD. Formação de recursos humanos na área da saúde do idoso. Rev Latino-am Enfermagem. 2004 Mar-Abr; 12(2):280-2. 